Article

\title{
A Facile Fabrication of Alginate Microbubbles Using a Gas Foaming Reaction
}

\author{
Keng-Shiang Huang ${ }^{1}$, Yung-Sheng Lin ${ }^{2}$, Wan-Ru Chang ${ }^{3}$, Yi-Ling Wang ${ }^{3}$ and Chih-Hui Yang ${ }^{3}{ }^{*}$ \\ 1 The School of Chinese Medicine for Post-Baccalaureate, I-Shou University, Kaohsiung 82445, \\ Taiwan; E-Mail: huangks@isu.edu.tw \\ 2 Department of Applied Cosmetology and Master Program of Cosmetic Science, \\ Hungkuang University, Taichung 43302, Taiwan; E-Mail: linys@sunrise.hk.edu.tw \\ 3 Department of Biological Science and Technology, I-Shou University, Kaohsiung 82445, Taiwan; \\ E-Mails: dale1511@hotmail.com (W.-R.C.); callingsummer@hotmail.com (Y.-L.W.) \\ * Author to whom correspondence should be addressed; E-Mail: chyang@isu.edu.tw; \\ Tel.: +886-7-615-1100 (ext. 7312); Fax: +886-7-657-7057.
}

Received: 17 July 2013; in revised form: 6 August 2013 / Accepted: 7 August 2013 /

Published: 12 August 2013

\begin{abstract}
Microbubble particles have been extensively utilized as temporal templates for various biomedical applications. This study proposes a facile strategy to obtain microbubble-containing alginate particles (i.e., microbubbles inside alginate gel particles, called alginate microbubbles). The chemical reaction of sodium bicarbonate and hydrogen peroxide to produce gaseous carbon dioxide and oxygen was utilized to form microbubbles within alginate particles. Uniform alginate particles were obtained by a stable needle-based droplet formation process. Kinetic reaction of gas formation was monitored for $2 \%$ alginate particles. The gas formation increased with the concentrations of sodium bicarbonate (1-5 wt $\%)$ and hydrogen peroxide (0-36.5 wt\%).
\end{abstract}

Keywords: microbubbles; alginate; gas; particle

\section{Introduction}

Porous biodegradable polymer substrates have extensive biomedical applications [1]. There has been a drastic increase in the use of microbubbles as vehicles for ultrasound-mediated imaging and targeted drug delivery [2-7]. Typical methods to fabricate porous structures include gas foaming [8], air 
pressure-driven injection [9], porogen elimination [10], emulsion/freeze drying [11,12], expansion in supercritical fluids [13], 3D ink-jet printing [14,15], etc. Some novel strategies to make microbubbles have been reviewed $[16,17]$. Among these methods, gas foaming based on a chemical reaction is one of the most simple and straightforward routes to form pores within a particle. It needs no extra equipment or instruments for porous structure generation. Besides being simple, low-cost, and easy to operate, chemical reactions have the added advantage of being effective in making gas pores [8].

Alginate is a natural polysaccharide derived from marine brown algae that finds numerous applications in diverse areas [18-20]. The two major fields of alginate applications are as biomedical devices for drug delivery and tissue engineering and as adsorbent materials for elimination of heavy metals and organic pollutants in water [21]. For these uses, exposed surface area is a crucial issue to determine its success. A high surface area will facilitate adsorption of water pollutants, but only suitable pore morphology can favor biomedical applications such as cell colonization. Alginate particles with porous inner structures have wide applications ranging from pharmaceuticals to foods and the cosmetic industry $[1,16,22]$. They have strong potential for molecular imaging, drug delivery, gene therapy, sonothrombolysis, and therapeutic treatment of antimicrobial films [23]. Calcium chloride-crosslinked gel-type alginate has a high viscosity, being capable of holding microbubbles for a long time [24]. These porous alginate particles can be applied as a contrast agent to enhance the ultrasound echo from the stomach and intestine through oral administration [24]. Furthermore, porous alginate particles can combine chemotherapeutic and imaging agents for ultrasound-mediated drug delivery because contrast-enhanced imaging provides for precise energy deposition [25].

Based on our previous works on the manufacture of uniform alginate particles [26-29], this work further develops a facile method to fabricate microbubble-containing alginate particles. The porous alginate particles (alginate microbubbles) prepared under different gas forming conditions were investigated and characterized. The manufactured alginate microbubbles provide great promise for biomedical applications such as chemotherapeutics and imaging.

\section{Results and Discussion}

\subsection{Ca-Alginate Microbubbles}

The stable needle-based droplet formation ensured a uniform particle size distribution. Figure 1 shows the collected Ca-alginate microbubbles in the receiving collector under the condition of $2 \%$ sodium bicarbonate and $18.25 \%$ hydrogen peroxide. As indicated in the pictures, the Ca-alginate particles are uniform in both morphology and size, and the average diameter is $2.12 \pm 0.15 \mathrm{~mm}$ (Figure 1A). In a close view (Figure 1B), gas development was randomly distributed within the alginate particle. The diameter of microbubbles reached several tens or hundreds micrometers and these pore sizes favor applications in cell colonization or metabolic waste removal, etc. [21]. The pore-closed surface may be due to the high viscosity of gel-type alginate that holds microbubbles entrapped in the particle [24].

\subsection{Microbubble Evolution}

Figure 2 indicates microbubble evolution in a kinetic process. The sodium bicarbonate is fixed at $1 \%$, and three hydrogen peroxide concentrations $(0 \%, 18.25 \%, 36.5 \%)$ are used for comparison. The 
predetermined five observation times are 30 seconds, $1 \mathrm{~min}, 3 \mathrm{~min}, 5 \mathrm{~min}$, and $10 \mathrm{~min}$, respectively. The photo image results show that the size and number of microbubbles increases with reaction time. Furthermore, microbubble development within an alginate particle is more conspicuous at a higher hydrogen peroxide concentration. The gas evolution after ten minutes is not significant (data not shown). Therefore, we collected porous alginate after alginate was immersed in hydrogen peroxide solution for ten minutes in the following study.

Figure 1. Photo images of porous alginate particles. (A) Uniform microparticle distribution with an average diameter of $2.25 \pm 0.11 \mathrm{~mm}$ and (B) Close-up view of a single microparticle with gas bubbles inside.

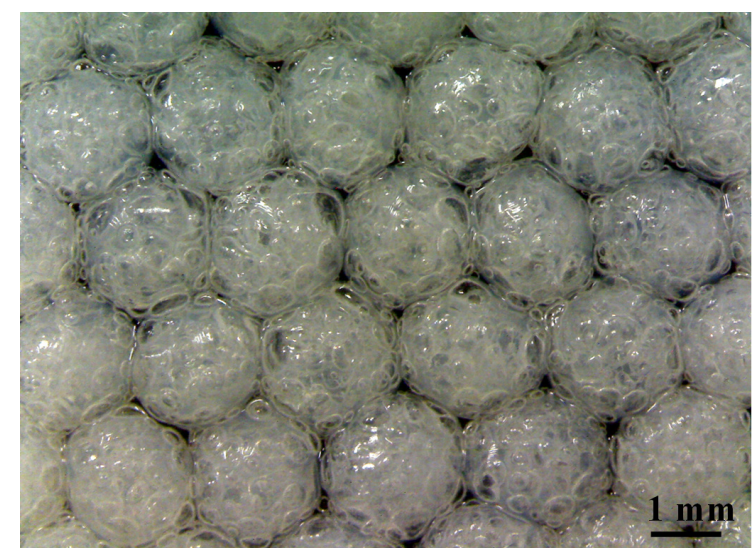

(A)

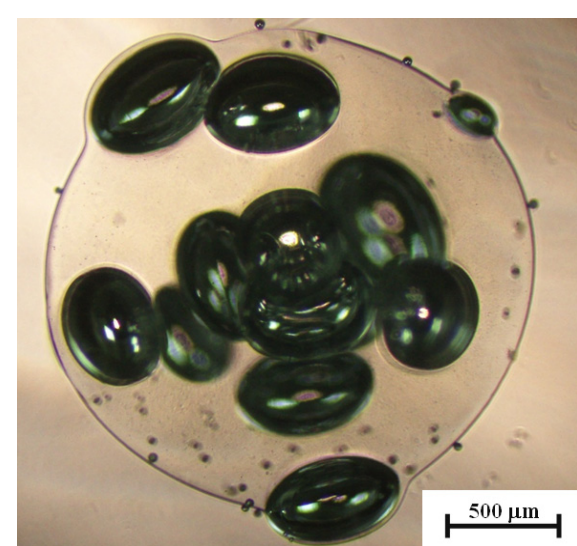

(B)

Figure 2. Photo images of the kinetics of gas formation in a single alginate microparticle. The concentration of $\mathrm{NaHCO}_{3}$ is fixed at $1 \%$ and the $\mathrm{H}_{2} \mathrm{O}_{2}$ concentrations are $0 \%(\mathbf{A}-\mathbf{E})$, $18.25 \%(\mathbf{F}-\mathbf{J})$, and $36.5 \%(\mathbf{K}-\mathbf{O})$. The gas formation time are 30 seconds (A, F, K), 1 minute $(\mathbf{B}, \mathbf{G}, \mathbf{L}), 3$ minutes $(\mathbf{C}, \mathbf{H}, \mathbf{M}), 5 \min (\mathbf{D}, \mathbf{I}, \mathbf{N})$, and $10 \min (\mathbf{E}, \mathbf{J}, \mathbf{O})$. Scale bar is $1 \mathrm{~mm}$.

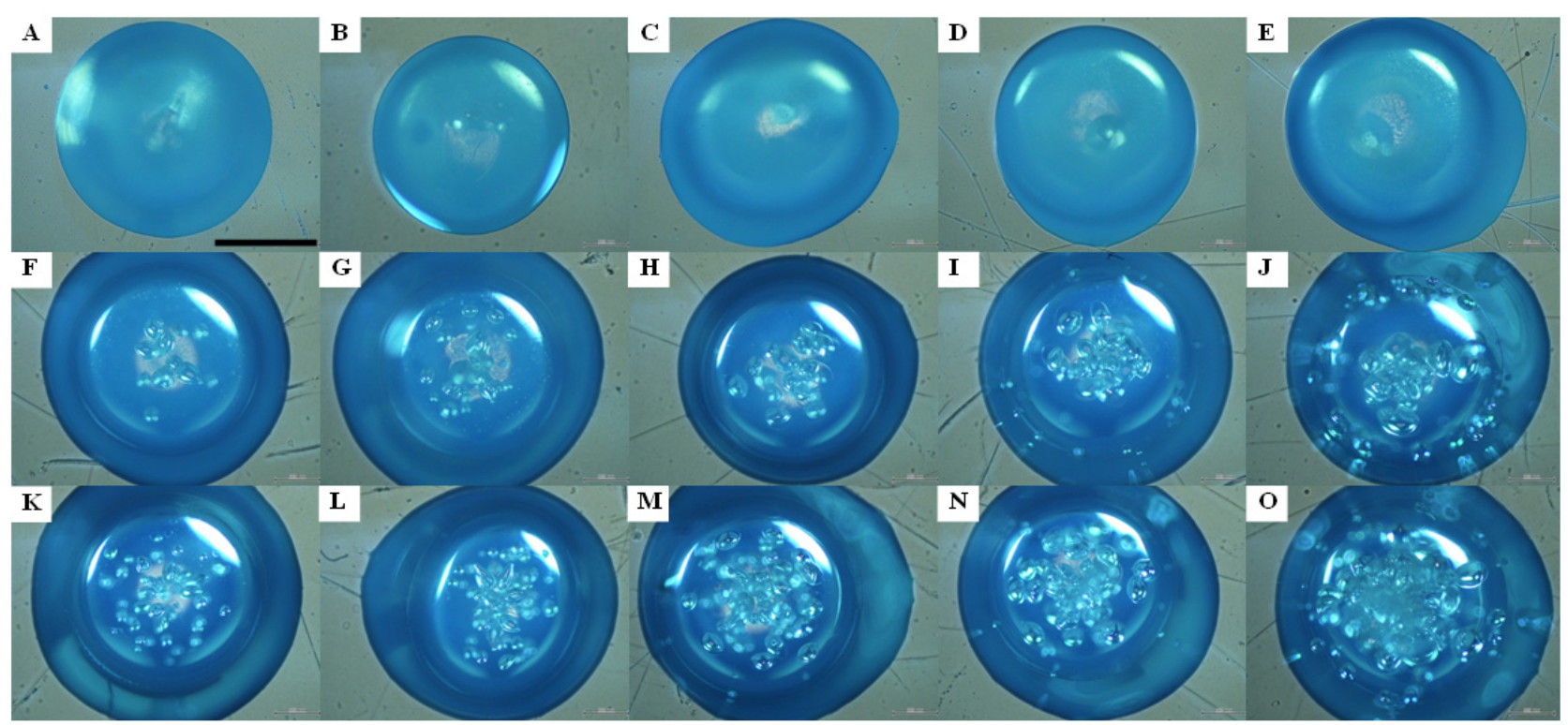


Figure 3 indicates the average size of microbubbles in the gas formation process. Results show that the there is no microbubble formation in the absence of hydrogen peroxide. Both the sodium bicarbonate and hydrogen peroxide are needed to produce the oxidative/reductive reaction that generates the gas. The size of microbubbles can reach about $400 \mu \mathrm{m}$. Furthermore, there is little difference between $18.25 \%$ and $36.5 \%$ hydrogen peroxide except in the early reaction stages. A smaller microbubbles size is formed in the early reaction phase for a higher hydrogen peroxide concentration. Due to the larger number of reactant molecules in $36.5 \%$ hydrogen peroxide, the number of reaction nuclei for each gas pore is larger. This results in a smaller microbubble size for a fixed sodium bicarbonate concentration.

Figure 3. Evolution of microbubble size in alginate particles under the conditions of Figure 2.

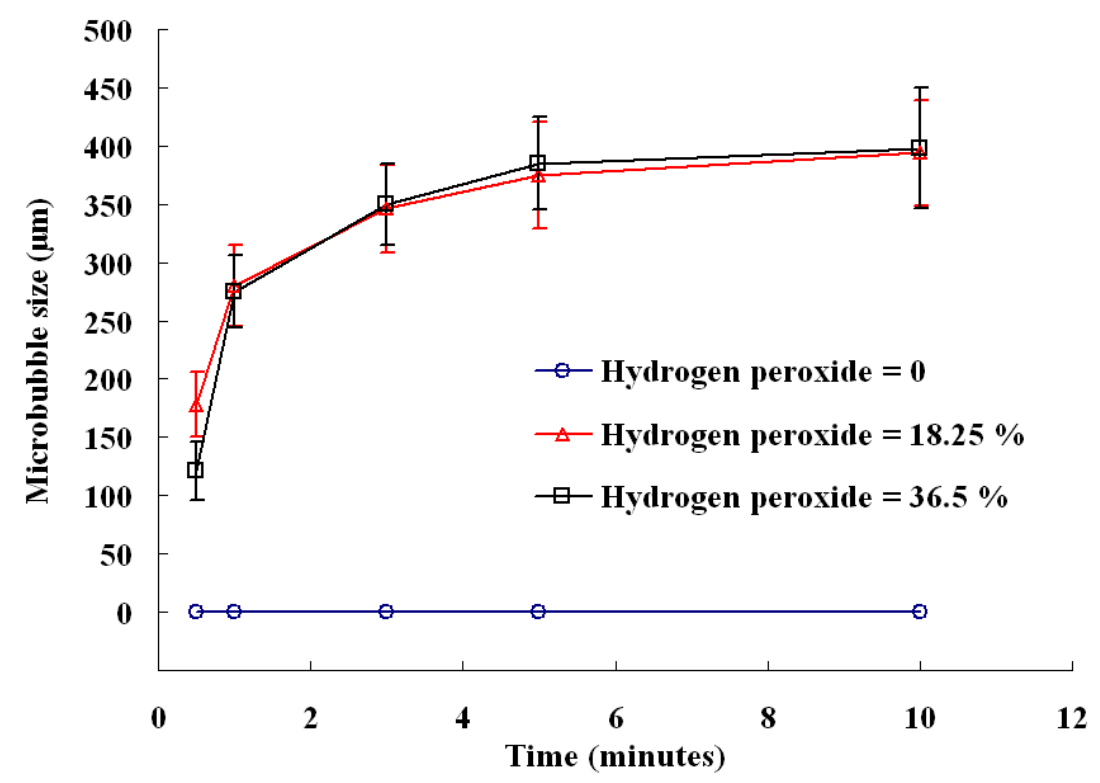

\subsection{Influence of Reactant Concentration}

Figures 4 and 5 investigate effects of sodium bicarbonate and hydrogen peroxide on the formation of microbubbles, respectively. To enhance the difference, the concentration of hydrogen peroxide is fixed at $36.5 \%$ in Figure 4, while sodium bicarbonate concentration is fixed at 5\% in Figure 5. Results reveal that gas formation increases with the concentration of sodium bicarbonate $(1 \%-5 \%$ in Figure 4$)$ and hydrogen peroxide $(0 \%-36.5 \%$ in Figure 5). Microbubble development within an alginate particle showed an enhancement for a high reactant concentration for both sodium bicarbonate and hydrogen peroxide. There is almost no gas formation in the absence of or with and $9.12 \%$ hydrogen peroxide (Figure 5).

Figure 4. Photo images of a single alginate microparticle prepared by a fixed $36.5 \% \mathrm{H}_{2} \mathrm{O}_{2}$ and different $\mathrm{NaHCO}_{3}$ concentrations: (A) 1\%, (B) 2\%, (C) 3\%, (D) 4\%, (E) 5\%. All scale bars are $1 \mathrm{~mm}$.
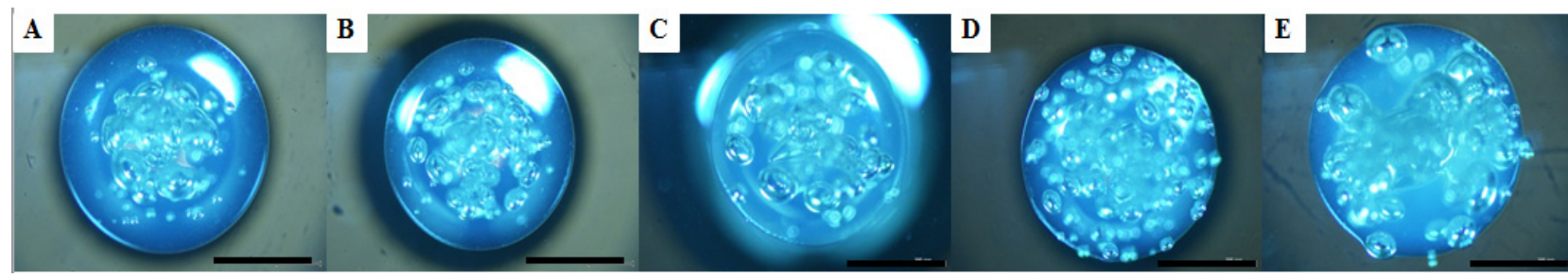
Figure 5. Photo images of a single alginate microparticle prepared by a fixed $5 \% \mathrm{NaHCO}_{3}$ and different $\mathrm{H}_{2} \mathrm{O}_{2}$ concentrations: (A) $0 \%$, (B) $9.12 \%$, (C) $18.25 \%$, (D) $27.37 \%$, (E) $36.5 \%$. All scale bars are $1 \mathrm{~mm}$.
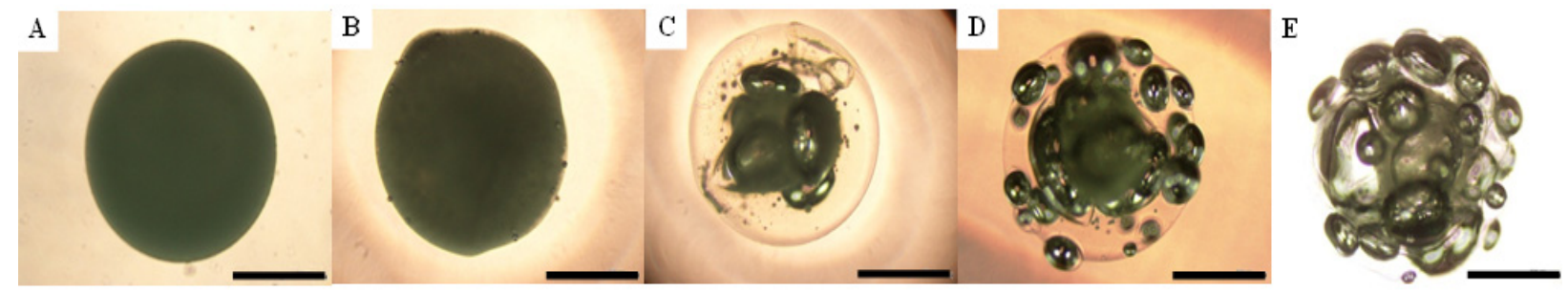

Due to the short diffusion path, the outer region of a particles showed an obvious gas formation, especially for a high reactant concentration. Most conventional contrast agents are vulnerable and lack stability [11]. Microbubbles are easily destroyed during the ultrasound diagnostic process. To have good detection quality, some constraints should be adopted, such as continuously infusing microbubbles, or lowering the ultrasound power, frame rate, and scan line density. Microbubbles composed of alginate have the advantage of eliminating these mentioned inconveniences. Alginate is a viscous hydrogel capable of holding microbubbles [24] for a good contrast agent efficacy. Besides, microbubble alginates can also carry chemotherapeutic agents for drug delivery applications.

Some novel microbubble preparation technologies are established. Microfluidic devices or other hydrodynamic atomization techniques can offer great control of microbubble size and polydispersity $[16,17,30]$. However these methods are usually cost-prohibitive, needing an extra gas source and equipment/instruments for microbubble generation. Besides, the production rate of microbubble particles is limited. Overcoming these inconveniences, the simple sodium bicarbonate-based gas formation proposed in this study offers the facile strategy to prepare microbubble particles. It has advantages of being simple, low-cost, and easy to operate. The gas formation reaction thus provides an alternative practical method in making gas pores [8].

\section{Experimental}

\subsection{Materials}

Alginic acid sodium salt (Na-alginate, brown algae with a viscosity $250 \mathrm{cP}$ in $2 \%(\mathrm{w} / \mathrm{v})$ solution at $25{ }^{\circ} \mathrm{C}$ ) and hydrogen peroxide $(36.5 \mathrm{wt} \%)$ were purchased from Sigma Chemical Co. (St. Louis, MO, USA). Calcium chloride and sodium bicarbonate (dehydrate, granular) were obtained from J.T. Baker Chemical Company (Phillipsburg, NJ, USA). All chemicals and solvents were of analytical reagent grade.

\subsection{Preparation of Ca-Alginate Particles}

As shown in Figure 6, Na-alginate solution loaded in a syringe (TERUMOR ${ }^{\circledR}$ Syringe, $3 \mathrm{~mL}$ ) was extruded from the needle tip $(24 \mathrm{G}, 0.55 \times 25 \mathrm{~mm}$ ) at a $0.01 \mathrm{~mL} / \mathrm{min}$ constant rate by a KDS230 syringe pump (KD Scientific Inc., Holliston, MA, USA). To generate microbubbles, sodium bicarbonate must be incorporated homogeneously with the Na-alginate solutions. The pendant Na-alginate solution at the tip of the needle was broken up to form a series of isolable Na-alginate droplets of about $2-3 \mathrm{~mm}$. The liquid in the receiving collector was filled with $25 \% \mathrm{w} / \mathrm{v}$ calcium chloride solution ( 2.5 grams calcium chloride in 
$10 \mathrm{~mL}$ hydrogen peroxide solution) for gelation. Sodium-alginate droplets are gelled in situ by immersion of $\mathrm{Ca}^{2+}$ ions for ten minutes, and then finally Ca-alginate particles were collected and characterized.

Figure 6. Schematic drawing of the experimental setup.

Syringe Pump

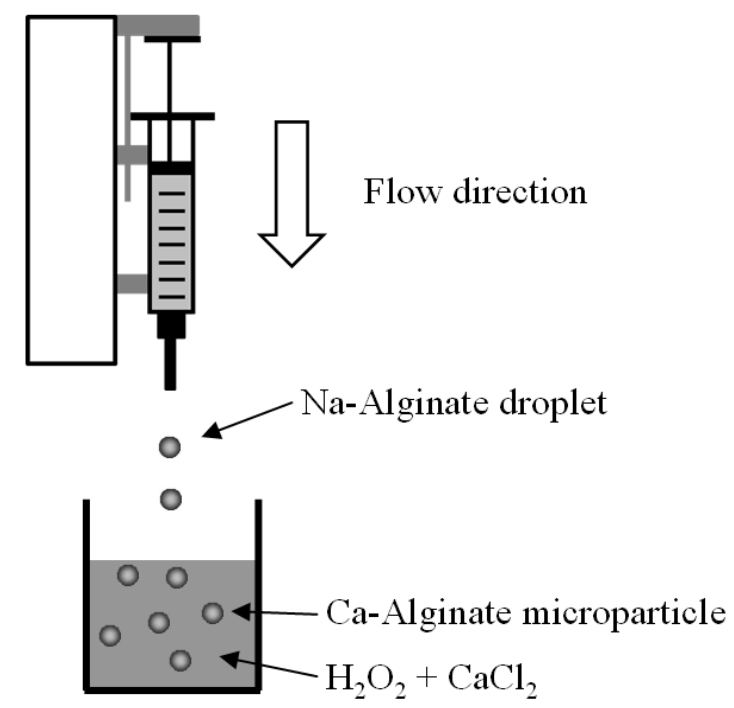

\subsection{Preparation of Ca-Alginate Microbubbles}

The gas formation within Ca-alginate particles can be illustrated by Figure 7. First of all, sodium bicarbonate was incorporated homogeneously in Na-alginate solutions. When the Na-alginate droplets were immersed in the receiving collector, hydrogen peroxide diffused inward into the droplet to react with sodium bicarbonate.

Figure 7. Schematic picture of structure evolution within a microparticle to form pores.

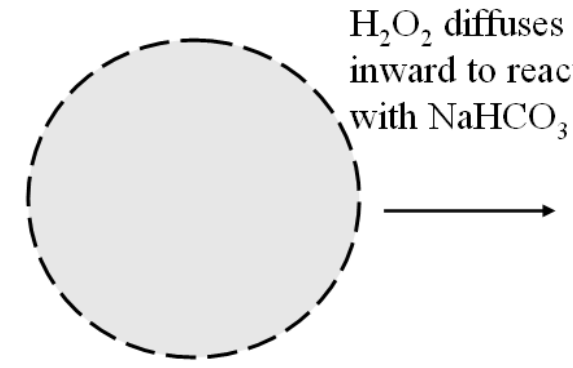

Alginate droplet containing $\mathrm{NaHCO}_{3}$

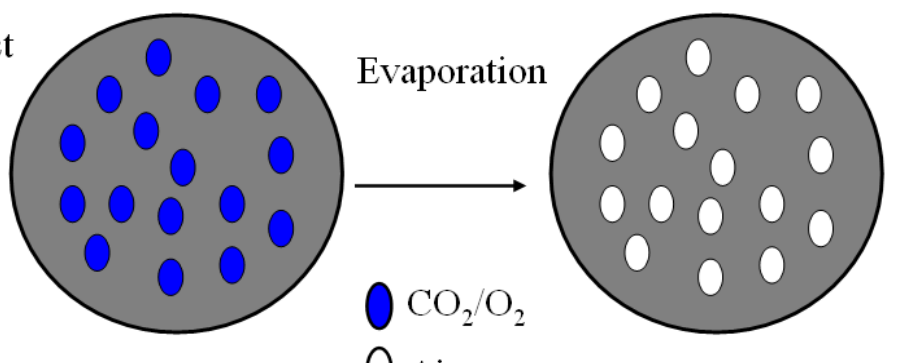

Air

Then carbon dioxide and oxygen gas can be generated in situ by an oxidative/reductive reaction between sodium bicarbonate and hydrogen peroxide. This process can be described by the following reactions [31]:

$$
\begin{gathered}
\mathrm{NaHCO}_{3}+\mathrm{H}_{2} \mathrm{O}_{2} \leftrightarrow \mathrm{NaHCO}_{4} \cdot \mathrm{H}_{2} \mathrm{O} \\
\mathrm{NaHCO}_{4} \cdot \mathrm{H}_{2} \mathrm{O} \rightarrow \mathrm{Na}_{2} \mathrm{CO}_{3} \cdot 1.5 \mathrm{H}_{2} \mathrm{O}_{2}+\mathrm{CO}_{2}+1.5 \mathrm{H}_{2} \mathrm{O}+0.25 \mathrm{O}_{2}
\end{gathered}
$$


After gas exchange with the surrounding air, the generated $\mathrm{CO}_{2}$ and $\mathrm{O}_{2}$ diffused out, and the microbubbles within the particle were finally filled with air.

\subsection{Characterization}

An optical microscope system (TE2000U, Nikon, Lewisville, TX, USA) and a digital camera (Evolution color VF, Media Cybernetics, Silver Spring, MD, USA) were utilized to observe the morphology of the collected particles. To ensure statistical representation, more than 50 particles were analyzed. The size of microbubbles was obtained from their photomicrographs and expressed as mean $\pm \mathrm{SD}$ (standard deviation).

\section{Conclusions}

Alginate microbubbles have great potential to combine chemotherapeutic and imaging agents for ultrasound-mediated drug delivery applications. Providing a cost-effective approach, this study successfully developed a sodium bicarbonate/hydrogen peroxide-based chemical reaction to generate porous alginate particles. The gas formation increased with the reactant concentration, and the gas reaction evolved in ten minutes for an alginate particle of $2 \mathrm{~mm}$ in diameter. This finding provides a facile strategy to prepare microbubble particles. This proposed method has the advantages of easy operation, low cost, and effective pore formation.

\section{Acknowledgements}

This work was financially supported by a grant from the National Science Council of Taiwan, R.O.C.

\section{Conflict of Interest}

The authors declare no conflict of interest.

\section{References}

1. Kim, T.K.; Yoon, J.J.; Lee, D.S.; Park, T.G. Gas foamed open porous biodegradable polymeric microspheres. Biomaterials 2006, 27, 152-159.

2. Dressaire, E.; Bee, R.; Bell, D.C.; Lips, A.; Stone, H.A. Interfacial polygonal nanopatterning of stable microbubbles. Science 2008, 320, 1198-1201.

3. Capece, S.; Chiessi, E.; Cavalli, R.; Giustetto, P.; Grishenkov, D.; Paradossi, G. A general strategy for obtaining biodegradable polymer shelled microbubbles as theranostic devices. Chem. Commun. 2013, 49, 5763-5765.

4. Hosny, N.A.; Mohamedi, G.; Rademeyer, P.; Owen, J.; Wu, Y.; Tang, M.X.; Eckersley, R.J.; Stride, E.; Kuimova, M.K. Mapping microbubble viscosity using fluorescence lifetime imaging of molecular rotors. Proc. Natl. Acad. Sci. USA 2013, 110, 9225-9230.

5. Geers, B.; de Wever, O.; Demeester, J.; Bracke, M.; de Smedt, S.C.; Lentacker, I. Targeted liposome-loaded microbubbles for cell-specific ultrasound-triggered drug delivery. Small 2013, doi:10.1002/smll.201300161. 
6. Noble, M.L.; Kuhr, C.S.; Graves, S.S.; Loeb, K.R.; Sun, S.S.; Keilman, G.W.; Morrison, K.P.; Paun, M.; Storb, R.F.; Miao, C.H. Ultrasound-targeted microbubble destruction-mediated gene delivery into canine livers. Mol. Ther. 2013, doi:10.1038/mt.2013.107.

7. Villa, R.; Cerroni, B.; Viganò, L.; Margheritelli, S.; Abolafio, G.; Oddo, L.; Paradossi, G.; Zaffaroni, N. Targeted doxorubicin delivery by chitosan-galactosylated modified polymer microbubbles to hepatocarcinoma cells. Colloids Surf. B Biointerfaces 2013, 110, 434-442.

8. Bae, S.E.; Son, J.S.; Park, K.; Han, D.K. Fabrication of covered porous PLGA microspheres using hydrogen peroxide for controlled drug delivery and regenerative medicine. J. Control. Release 2009, 133, 37-43.

9. Wang, X.L.; Li, X.; Stride, E.; Huang, J.; Edirisinghe, M.; Schroeder, C.; Best, S.; Cameron, R.; Waller, D.; Donald, A. Novel preparation and characterization of porous alginate films. Carbohyd. Polym. 2010, 79, 989-997.

10. Chevalier, E.; Chulia, D.; Pouget, C.; Viana, M. Fabrication of porous substrates: A review of processes using pore forming agents in the biomaterial field. J. Pharm. Sci. 2008, 97, 1135-1154.

11. Cui, W.; Bei, J.; Wang, S.; Zhi, G.; Zhao, Y.; Zhou, X.; Zhang, H.; Xu, Y. Preparation and evaluation of poly(L-lactide-co-glycolide) (PLGA) microbubbles as a contrast agent for myocardial contrast echocardiography. J. Biomed. Mater. Res. B Appl. Biomater. 2005, 73, 171-178.

12. Zhang, H.; Ju, X.J.; Xie, R.; Cheng, C.J.; Ren, P.W.; Chu, L.Y. A microfluidic approach to fabricate monodisperse hollow or porous poly(HEMA-MMA) microspheres using single emulsions as templates. J. Colloid Interface Sci. 2009, 336, 235-243.

13. Butler, R.; Davies, C.M.; Cooper, A.I. Emulsion templating using high internal phase supercritical fluid emulsions. Adv. Mater. 2001, 13, 1459-1463.

14. Yang, S.; Leong, K.F.; Du, Z.; Chua, C.K. The design of scaffolds for use in tissue engineering. Part II. Rapid prototyping techniques. Tissue Eng. 2002, 8, 1-11.

15. Tsang, V.L.; Bhatia, S.N. Three-dimensional tissue fabrication. Adv. Drug Deliv. Rev. 2004, 56, 1635-1647.

16. Stride, E.; Edirisinghe, M. Novel microbubble preparation technologies. Soft Matter 2008, 4, 2350-2359.

17. Stride, E.; Edirisinghe, M. Novel preparation techniques for controlling microbubble uniformity: A comparison. Med. Biol. Eng. Comput. 2009, 47, 883-892.

18. Coppi, G.; Iannuccelli, V. Alginate/chitosan microparticles for tamoxifen delivery to the lymphatic system. Int. J. Pharm. 2009, 367, 127-132.

19. Chen, C.C.; Fang, C.L.; Al-Suwayeh, S.A.; Leu, Y.L.; Fang, J.Y. Transdermal delivery of selegiline from alginate-Pluronic composite thermogels. Int. J. Pharm. 2011, 415, 119-128.

20. Balaure, P.C.; Andronescu, E.; Grumezescu, A.M.; Ficai, A.; Huang, K.S.; Yang, C.H.; Chifiriuc, C.M.; Lin, Y.S. Fabrication, characterization and in vitro profile based interaction with eukaryotic and prokaryotic cells of alginate-chitosan-silica biocomposite. Int. J. Pharm. 2013, 441, 555-561.

21. Barbetta, A.; Barigelli, E.; Dentini, M. Porous alginate hydrogels: Synthetic methods for tailoring the porous texture. Biomacromolecules 2009, 10, 2328-2337. 
22. Wan, J.; Bick, A.; Sullivan, M.; Stone, H.A. Controllable microfluidic production of microbubbles in water-in-oil emulsions and the formation of porous microparticles. Adv. Mater. 2008, 20, 3314-3318.

23. Cavalieri, F.; Zhou, M.; Tortora, M.; Lucilla, B.; Ashokkumar, M. Methods of preparation of multifunctional microbubbles and their in vitro/in vivo assessment of stability, functional and structural properties. Curr. Pharm. Des. 2012, 18, 2135-2151.

24. Soetanto, K.; Chan, M.; Okujima, M. Effect of calcium chloride on sodium alginate microbubbles as ultrasound contrast agent. Jpn. J. Appl. Phys. 1996, 35, 3152-3155.

25. Sridhar-Keralapura, M.; Thirumalai, S.; Mobed-Miremadi, M. Structural changes and imaging signatures of acoustically sensitive microcapsules under ultrasound. Ultrasonics 2013, 53, 1044-1057.

26. Huang, K.S.; Yang, C.H.; Lin, Y.S.; Wang, C.Y.; Lu, K.; Chang, Y.F.; Wang, Y.L. Electrostatic droplets assisted synthesis of alginate microcapsules. Drug Deliv. Transl. Res. 2011, 1, 289-298.

27. Huang, K.S.; Lin, Y.S.; Yang, C.H.; Tsai, C.W.; Hsu, M.Y. In situ synthesis of twin monodispersed alginate microparticles. Soft Matter 2011, 7, 6713-6718.

28. Wang, C.Y.; Yang, C.H.; Lin, Y.S.; Chen, C.H.; Huang, K.S. Anti-inflammatory effect with high intensity focused ultrasound-mediated pulsatile delivery of diclofenac. Biomaterials 2012, 33, $1547-1553$.

29. Lin, Y.S.; Yang, C.H.; Hsu, Y.Y.; Hsieh, C.L. Microfluidic synthesis of tail-shaped alginate microparticles using slow sedimentation. Electrophoresis 2013, 34, 425-431.

30. Gong, X.; Wen, W.; Sheng, P. Microfluidic fabrication of porous polymer microspheres: Dual reactions in single droplets. Langmuir 2009, 25, 7072-7077.

31. Firsova, T.P.; Sokol, V.I.; Bakulina, V.M.; Stasevich, N.N. Reaction of sodium bicarbonate with hydrogen peroxide and some properties of the compound $\mathrm{Na}_{2} \mathrm{CO}_{3} 1.5 \mathrm{H}_{2} \mathrm{O}_{2}$. Russ. Chem. Bull. 1968, 17, 1850-1853.

Sample Availability: Not available.

(C) 2013 by the authors; licensee MDPI, Basel, Switzerland. This article is an open access article distributed under the terms and conditions of the Creative Commons Attribution license (http://creativecommons.org/licenses/by/3.0/). 